\title{
INITIAL ASSESSMENT OF THE PERFORMANCE OF THE FIRST WIND LIDAR IN SPACE ON AEOLUS
}

\author{
Oliver Reitebuch ${ }^{1}$, Christian Lemmerz ${ }^{1}$, Oliver Lux ${ }^{1}$, Uwe Marksteiner ${ }^{1}$, Stephan Rahm ${ }^{1}$, \\ Fabian Weiler ${ }^{1}$, Benjamin Witschas ${ }^{1}$, Markus Meringer ${ }^{2}$, Karsten Schmidt ${ }^{2}$, Dorit Huber ${ }^{3}$, \\ Ines Nikolaus $^{4}$, Alexander Geiss ${ }^{5}$, Michael Vaughan ${ }^{6}$, Alain Dabas ${ }^{7}$, Thomas Flament ${ }^{7}$, \\ Hugo Stieglitz ${ }^{7}$, Lars Isaksen ${ }^{8}$, Michael Rennie ${ }^{8}$, Jos de Kloe', Gert-Jan Marseille', Ad Stoffelen', \\ Denny Wernham ${ }^{10}$, Thomas Kanitz ${ }^{10}$, Anne-Grete Straume ${ }^{10}$, Thorsten Fehr ${ }^{10}$, \\ Jonas von Bismarck ${ }^{11}$, Rune Floberghagen ${ }^{11}$, Tommaso Parrinello ${ }^{11}$ \\ ${ }^{I}$ DLR, Institute of Atmospheric Physics, Oberpfaffenhofen, Germany \\ ${ }^{2}$ DLR, Remote Sensing Technology Institute, Oberpfaffenhofen, Germany \\ ${ }^{3}$ DoRIT, Fürstenfeldbruck, Germany \\ ${ }^{4}$ University of Applied Sciences, Munich, Germany \\ ${ }^{5}$ Ludwig-Maximillians-University, Meteorological Institute, Munich, Germany \\ ${ }^{6}$ Optical \& Lidar Associates OLA, Buckinghamshire, United Kingdom \\ ${ }^{7}$ Météo-France, CNRM, Toulouse, France \\ ${ }^{8} E C M W F$, Reading, United Kingdom \\ ${ }^{9} \mathrm{KNMI}$, de Bilt, The Netherlands \\ ${ }^{10}$ ESA-ESTEC, Noordwijk, The Netherlands \\ ${ }^{11}$ ESA-ESRIN, Frascati, Italy \\ *Email: oliver.reitebuch@dlr.de
}

\begin{abstract}
Soon after its successful launch in August 2018, the spaceborne wind lidar ALADIN (Atmospheric LAser Doppler INstrument) on-board ESA's Earth Explorer satellite Aeolus has demonstrated to provide atmospheric wind profiles on a global scale. Being the first ever Doppler Wind Lidar (DWL) instrument in space, ALADIN contributes to the improvement in numerical weather prediction (NWP) by measuring one component of the horizontal wind vector. The performance of the ALADIN instrument was assessed by a team from ESA, DLR, industry, and NWP centers during the first months of operation. The current knowledge about the main contributors to the random and systematic errors from the instrument will be discussed. First validation results from an airborne campaign with two wind lidars on-board the DLR Falcon aircraft will be shown.
\end{abstract}

\section{INTRODUCTION}

The Aeolus satellite was successfully launched from Kourou (French Guiana) on August 22, 2018. Its single payload ALADIN is the first European lidar and the first DWL in space [1]. It is considered as a technology demonstrator for future operational wind lidar missions. Aeolus provides profiles of one component of the horizontal wind vector along the laser line-ofsight (LOS, projected to the horizontal HLOS) from ground up to the lower stratosphere $(20 \mathrm{~km}$ to $30 \mathrm{~km}$ ) with $250 \mathrm{~m}$ to $2 \mathrm{~km}$ vertical resolution. A wind profile is obtained from horizontal averaging along track in order to achieve the precision requirement (random error) of $1 \mathrm{~m} / \mathrm{s}$ to $2.5 \mathrm{~m} / \mathrm{s}$ (HLOS) depending on altitude [2]. The horizontal averaging length of typically $90 \mathrm{~km}$ might vary depending on signal-to-noise ratio (SNR) and atmospheric scene. Systematic errors which cannot be corrected by a-priori knowledge (e.g., calibration or instrumental corrections) also called "unknown biases" - are very detrimental for NWP. Thus, the requirement for the maximum bias of $0.7 \mathrm{~m} / \mathrm{s}$ (HLOS) is more stringent than the random error requirement.

\section{THE ALADIN LIDAR}

The lidar ALADIN is based on a direct-detection DWL operating at an ultraviolet (UV) wavelength of $354.8 \mathrm{~nm}$ [3]. The optical receiver consists of two spectrometers to determine the Doppler shift from the spectrally broad Rayleigh-Brillouin molecular backscatter and the spectrally narrow Mie backscatter from aerosols and cloud particles. Accumulation charge coupled device (ACCD) 
detectors are used for both the Mie and Rayleigh spectrometer. The minimum vertical resolution of the ALADIN instrument is limited by the ACCD detectors to $250 \mathrm{~m}$ and 24 different altitude levels (range bins). The vertical resolution can be varied between $250 \mathrm{~m}$ and $2000 \mathrm{~m}$ within one vertical profile. The number of laser pulse returns accumulated directly on the detector is a configurable parameter and determines the minimum along-track horizontal resolution for the raw data, which is typically $3 \mathrm{~km}$ for a number of 20 accumulated laser pulses (called "measurement"). Both the Mie and the Rayleigh spectrometer data are stored with this temporal resolution on-board. Further averaging of 600 laser pulse returns to so-called "observations" is performed on-ground for the Level $1 \mathrm{~b}$ (L1b) products [4]. A grouping algorithm is applied for Level $2 b$ (L2b) wind products which classifies the measurements in clear air Rayleigh winds and cloudy Mie winds [5]. The L2b Rayleigh wind retrieval uses temperature and pressure profiles from NWP models for taking into account the influence onto the molecular lineshape broadening arising from Rayleigh-Brillouin scattering [6].

\section{RESULTS}

Only nine days after launch on August $31^{\text {st }}$ ALADIN was switched-on and first backscattered light from the atmosphere was sampled on September $5^{\text {th }}$ showing typical lidar signals including molecular returns, cloud tops and extinction below the clouds (Fig. 1). The first atmospheric Doppler frequency shifts and atmospheric winds could be retrieved already three weeks after launch [7] and first results regarding the performance were reported during ICSO in October 2018 [8].

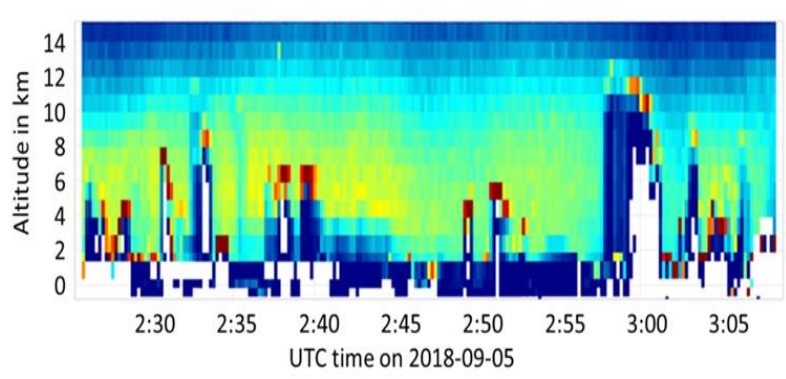

Figure 1 First atmospheric signal from the Rayleigh spectrometer received on 5 September 2018 along 1/2 orbit (visualized with VirES[9])

\subsection{Random error performance}

The random errors for ALADIN are mainly influenced by SNR and spectral widths of the backscattered signals. The dominating noise source for both Mie and Rayleigh spectrometer winds is arising from signal photon shot noise. The electronic noise in the detection chain is significantly lower due to the accumulation principle of the ACCD allowing photon counting. Apart from the range of the satellite to the atmosphere and the range resolution, the detected signal levels are mainly determined by both the emitted energy as well as the optical receive path transmission.

The evolution of the laser energy from the first switch-on until mid-January 2019, where the ALADIN instrument went into a standby mode, is shown in Fig. 2. A maximum energy of $65 \mathrm{~mJ}$ (UV) was measured initially which decreased to $50 \mathrm{~mJ}$ until mid-January, hence, being lower than the anticipated $80 \mathrm{~mJ}$. By modifying the operating parameters (e.g. temperatures or currents) during the first months in order to increase the emitted laser energy, both the UV and IR energies were affected. A stable IR energy level was achieved by mid-December, which might indicate a thermo-mechanical settling in the laser. It was demonstrated that a UV laser could be operated in space over several months proving the successful deployment of an oxygen cleaning system to prevent laser induced contamination.

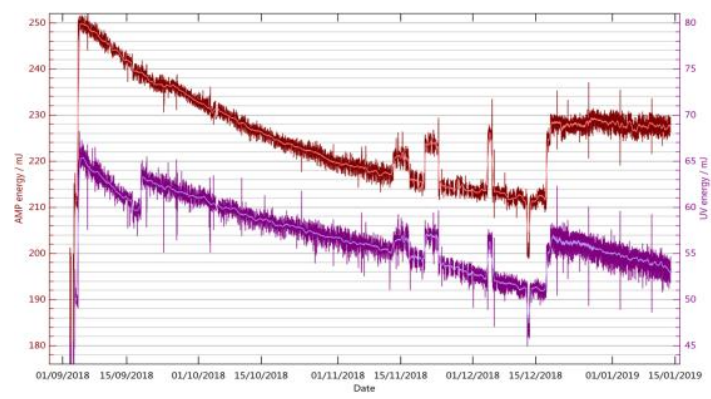

Figure 2 Evolution of laser energy from initial switchon in September 2018 until mid-January 2019.

As the atmospheric molecular Rayleigh backscatter for high altitudes and above clouds depends only on atmospheric density and hence atmospheric temperature and pressure, the signal levels (i.e. the radiometric performance) were first assessed using the Rayleigh spectrometer signals. 
Comparison of signal levels using an end-to-end simulator (E2S) with measured atmospheric signals showed lower signals by a factor of 2.5 to 3 consistently for different observing geometries (off-nadir, nadir pointing), over one orbit and over the period from September to December. A factor of 1.25 to 1.6 can be explained with lower laser energy, as the pre-launch simulations were performed with $80 \mathrm{~mJ}$ and only $50 \mathrm{~mJ}$ to $65 \mathrm{~mJ}$ were achieved in-orbit. A factor of 1.5 to 2 is presumably arising from further signal losses in the optical transmit-receive path. It is hereby assumed that signal clipping at the instrument field stop occurs, which limits the field-of-view to only $18 \mu \mathrm{rad}$. This leads to an increase of the Rayleigh wind errors from $2.5 \mathrm{~m} / \mathrm{s}$ (expected prelaunch with $80 \mathrm{~mJ}$ ) to about $4 \mathrm{~m} / \mathrm{s}$, which is confirmed by comparison with NWP models.
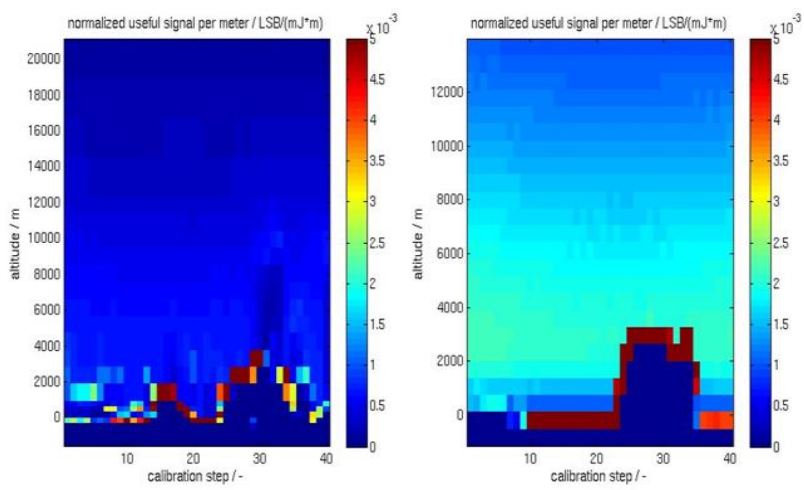

Figure 3 Comparison of normalized Rayleigh useful signal (least significant bit LSB/mJ m) from observations (left) and E2S (right) over Antarctica in nadir-pointing during a response calibration (step\# corresponds to distance); high signals (red colors) show ground-return signals over ice.

\subsection{Systematic errors}

Already in mid-September 2018, a systematic error in Rayleigh winds for one specific range gate was spotted by comparison of several orbits of Aeolus data with the ECMWF model. This bias in Rayleigh winds could be traced back to one suspicious pixel on the Rayleigh ACCD which showed enhanced dark current levels compared to the other pixels by only a few digitizer counts. The number of these "hot" pixels increased continuously over time affecting five range gates for Mie and five range gates for Rayleigh winds (as of mid-March 2019). A specific instrument mode to characterize the dark current levels and occurrence of the "hot" pixels was introduced, and correction algorithms are currently tested.

Furthermore, slow drifts of the incidence angles on the spectrometers were observed which lead to slow drifts in the systematic errors. A strategy for correcting these drifts is currently being refined. It will be based on a combination of spectral characterization using internal measurements, response calibrations performed in nadir pointing, and the utilization of ground-return velocities to correct for remaining linear and harmonic drifts in wind speed bias.

\subsection{First airborne validation results}

Already several years before the launch, an airborne prototype of the Aeolus payload, the ALADIN Airborne Demonstrator (A2D), was developed at DLR. Owing to its representative design and operating principle, the A2D provides valuable information on the wind measurement strategies of the satellite instrument as well as on the optimization of the wind retrieval and related quality-control algorithms [10, 11]. In November 2018, the first airborne validation campaign after launch was carried out from the airbase in Oberpfaffenhofen, Germany. Four underflights of the Aeolus satellite could be realized and provided first comparisons between the two airborne wind lidars (A2D and a coherent 2- $\mu \mathrm{m}$ wind lidar) and the satellite instrument (Fig. 4).

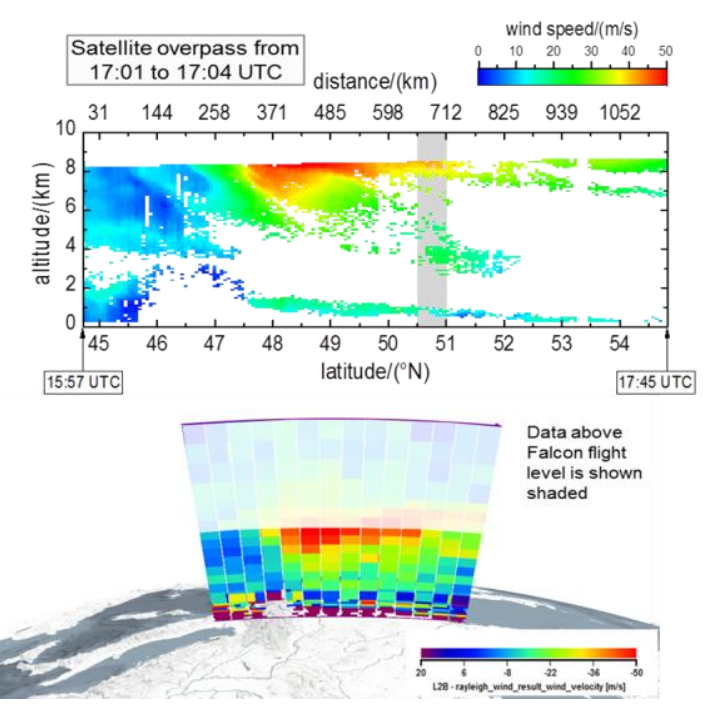

Figure 4 First airborne validation of L2b Rayleigh winds with 2- $m$ wind lidar (top) from 17 November 2018. Aeolus data (bottom) is visualized by VirES [9]. 


\section{CONCLUSIONS}

From a lidar-instrument perspective the ALADIN measurements of the first half year have demonstrated that a space-borne wind lidar can measure atmospheric winds by analyzing molecular Rayleigh and cloud Mie backscatter. It has been shown that a powerful UV laser can be operated in space with high frequency stability. This particularly proves the approach of using oxygen cleaning for mitigation the risk of LIC on optics in the UV for the first time.

The Rayleigh wind random errors are about $4 \mathrm{~m} / \mathrm{s}$ and therefore higher than the expected value of $2.5 \mathrm{~m} / \mathrm{s}$ from pre-launch simulations. This is mainly due to the significantly lower Rayleigh signal levels (factor 2.5-3) due to enhanced signal losses in the optical receiver (presumably at the field stop) and the lower laser energy between $50 \mathrm{~mJ}$ and $65 \mathrm{~mJ}$ compared to the target of $80 \mathrm{~mJ}$. As it could be demonstrated, that no significant additional noise sources are present, the expected gain in signal would be a factor of 1.6 with $80 \mathrm{~mJ}$, which is anticipated for the redundant laser.

The ongoing characterization of instrumental drifts and calibration will allow correction for the known biases with the upcoming algorithm, processor and data products updates. Correction for the enhanced dark signal levels of some ACCD pixels are currently being implemented and will almost completely remove the biases observed for single range gates for both the Mie and Rayleigh spectrometer output. A strategy for correction of instrumental drifts is being refined, based on a combination of spectral characterization using internal measurements, response calibrations performed in nadir pointing, and the use of ground-return velocities to correct for remaining linear and harmonic drifts in wind speed bias.

\section{ACKNOWLEDGEMENTS}

This work was supported by ESA under contract no. 18366/04/NL/MM and the Aeolus DISC contract no. 40000126336/18/I-BG. The airborne campaigns were funded by DLR and ESA under contract no 4000114053/15/NL/FF/gp.

\section{REFERENCES}

[1] European Space Agency ESA, 2008: ADMAeolus Science Report, ESA SP-1311, 121 pp.

[2] European Space Agency ESA, 2016: ADMAeolus Mission Requirements Documents, AERP-ESA-SY-001, Issue 2, 16/11/2016, 57pp.

[3] Reitebuch, O., 2012: The space-borne wind lidar mission ADM-Aeolus. in Atmospheric Physics Background, Methods, Trends. U. Schumann (Ed.) Springer Series on Research Topics in Aerospace, 815-827.

[4] DLR: Reitebuch, O., Huber, D., Nikolaus, I., (2018). Algorithm Theoretical Basis Document ATBD: ADM-Aeolus Level 1B Products, AE-RPDLR-L1B-001, V. 4.4, 20.04.2018, 117pp.

[5] ECMWF: Tan, D., M. Rennie, E. Andersson, P. Poli, A. Dabas, J. de Kloe, Gert-Jan Marseille, Ad Stoffelen 2018: ADM-Aeolus Level-2B Algorithm Theoretical Baseline Document. AE-TNECMWF-L2BP-0024, Version 3.1, 31 Jan 2018, 133 pages.

[6] Dabas, A., Denneulin, M.-L., Flamant, P., Loth, C., Garnier, A., Dolfi-Bouteyre, A., 2008: Correcting winds measured with a Rayleigh Doppler lidar from pressure and temperature effects. Tellus 60A, 206-215.

[7] ESA 2018: Aeolus wows with first wind data, press release on 12 September 2018 https://www.esa.int/Our_Activities/Observing the Earth/Aeolus/

[8] Kanitz, T. J. Lochard, J Marshall, P. McGoldrick, O. Lecrenier, P. Bravetti, O. Reitebuch, M. Rennie, D. Wernham, and A. Elfving, 2018: Aeolus First Light - First Glimpse. Proc. Int. Conf. Space Optics ICSO, 9-12 October 2018, Chania, Greece.

[9] VirES for Aeolus 2018: https://aeolus.services/

[10] Lux, O., Lemmerz, C., Weiler, F., Marksteiner, U., Witschas, B., Rahm, S., Schäfler, A., Reitebuch, O. 2018: Airborne wind lidar observations over the North Atlantic in 2016 for the pre-launch validation of the satellite mission Aeolus. Atmos. Meas. Tech., 3297-3322.

[11] Marksteiner, U., Ch. Lemmerz, O. Lux, S. Rahm, A. Schäfler , B. Witschas, O. Reitebuch 2018: Calibrations and Wind Observations of an Airborne Direct-Detection Wind LiDAR Supporting ESA's Aeolus Mission. Remote Sensing, 10, 2056. 